\title{
Identification of image attributes that are most affected with changes in displayed image size
}

\author{
Jae Young Park, Sophie Triantaphillidou and Ralph E. Jacobson \\ University of Westminster, Watford Road, Harrow, HA1 3PT, UK
}

\begin{abstract}
This paper describes an investigation of changes in image appearance when images are viewed at different image sizes on a high-end LCD device. Two digital image capturing devices of different overall image quality were used for recording identical natural scenes with a variety of pictorial contents. From each capturing device, a total of sixty four captured scenes, including architecture, nature, portraits, still and moving objects and artworks under various illumination conditions and recorded noise level were selected. The test set included some images where camera shake was purposefully introduced. An achromatic version of the image set that contained only lightness information was obtained by processing the captured images in CIELAB space. Rank order experiments were carried out to determine which image attribute(s) were most affected when the displayed image size was altered. These evaluations were carried out for both chromatic and achromatic versions of the stimuli. For the achromatic stimuli, attributes such as contrast, brightness, sharpness and noisiness were rank-ordered by the observers in terms of the degree of change. The same attributes, as well as hue and colourfulness, were investigated for the chromatic versions of the stimuli. Results showed that sharpness and contrast were the two most affected attributes with changes in displayed image size. The ranking of the remaining attributes varied with image content and illumination conditions. Further, experiments were carried out to link original scene content to the attributes that changed mostly with changes in image size.
\end{abstract}

Keywords: Image appearance, image quality, image size, scene content, scene analysis

\section{INTRODUCTION}

Various display technologies are available in mobile devices (e.g. digital camera, mobile phones, etc), yet LCDs are the most common displays in mobile devices with digital image capturing functions. Since often the quality of images on small LCD devices is satisfactory, the users check the captured images and tend to believe that the images they see on the small display is what they will get when their images will be displayed on larger displays. However, the images displayed on small display devices do not give full information, since image quality is highly dependent on the physical properties of the images [1]. Due to the properties and sizes of the built-in LCDs in capturing devices, the physical properties of the images maybe affected and give distorted information.

Image quality involves the assessment of many perceptual attributes such as image contrast, resolution, sharpness, graininess and colour [1]. Until recently, studies on the perceived quality of display devices were based on physical measurements, carried out to investigate one or more quality attributes rather than the overall visual appearance of the displayed images [2, 3, 4, 5]. Images are increasingly displayed and viewed in various sizes, under various viewing conditions. The subjective impressions of image quality will thus be affected by changes in image size [6], scene content (or complexity) [1] and also by environmental conditions, such as background colours and the intensity and colour of surrounds [7, 8]. Nevertheless, traditionally display characterisation techniques do not account for the displayed image size, or scene content.

In recent years there has been several studies investigating the effect of displayed image size on colour appearance and/or image quality [7, 8, 9]. In colour appearance investigations by Choi et al, the studies were mainly focused on changes of the size of uniform patches, as well as illumination level, surround and relative display luminance. Measurements in these studies were restricted to a certain area of the display, with a small coverage under certain viewing conditions. Further, Nezamabadi et al [9] investigated the relationship between changes in image size and 
perceived contrast, using contrast matching techniques and artificially generated noise patterns of different spatial frequency content. In another study, Nezamabadi et al [8] investigated changes in perceived lightness and chroma with changes in visual angle and thus perceived image size. Spatial effects such as sharpness, noisiness and most importantly the appearance of digital image artefacts caused by varying image size, image content and illumination conditions have not been considered in depth. The work reported here has been carried out to identify those image attributes (both colour and spatial) that are most affected with changes in displayed image size. The experiment presented in this paper is part of a larger project which seeks to identify the degrees of change in image appearance by varying image size, pictorial content, whilst looking not only colour but further into spatial image quality attributes such as sharpness, noisiness and the appearance of digital image artefacts.

\section{TEST STIMULI PREPARATION}

\subsection{Image capture}

Two digital image capturing devices (with built-in LCDs) of different overall image quality were selected. An eight megapixel Canon 30D digital SLR camera, equipped with an EF-S10-22mm lens and a two megapixel Apple iPhone camera with a built-in lens were used for capturing the test images. For each captured scene both devices were set to the same focal length to record identical image frames. Because it was difficult to adjust shutter speed and aperture on the camera of Apple iPhone, several exposures were made with the Canon 30D in manual mode to match the captured image by the Apple iPhone camera. The most similarly exposed images by both cameras were selected by inspection to create an appropriate test set.

\subsection{Image selection}

Images were carefully selected to cover various possible situations and conditions captured by ordinary users. For each capturing device, a total of sixty four captured scenes including architectures, nature, portraits, still and moving objects and artworks under various illumination conditions with differing scene contrast and recorded noise levels were selected. The test sets also included some images where camera shake was purposefully introduced.

\subsection{Image processing}

The original captured images were too large to be displayed in full resolutions on the EIZO ColorEdge CG210 21.3” LCD which was used in this investigation. Thus, the test images were resized from their original sizes to 744 (v) $\mathrm{x} 560$ (h) pixels using bi-cubic interpolation. This size is approximately the half of the LCD's horizontal resolution and is an appropriate image size to be displayed on commonly used XGA displays. In order to obtain the achromatic versions of the test stimuli, all resized images were then converted from RGB to CIELAB space and the lightness channel ( ${ }^{*}$ ) was selected for the purpose.

\section{PSYCHOPHYSICAL INVESTIGATION}

\subsection{Calibration and settings of the system}

An EIZO ColorEdge CG210 LCD, driven by a Sony VAIO VGN-T92S computer with an on-board graphics controller was used in the psychophysical investigation. The LCD has a native resolution of 1,600 x 1,200 pixels (24 bits). The system was set to a white point luminance of $134.3 \mathrm{~cd} / \mathrm{m}^{2}$, a gamma of 2.2 and a colour temperature of $6500 \mathrm{~K}$, using the GretagMacbeth Eye-One Pro with Profilemaker5. 


\subsection{Software preparation and interface design}

The application employed in this rank order psychophysical experiment was written in JavaScript. It was tested and optimised in Mozilla Firefox v3.0.1 web browser. A mid-grey background colour at a gamma of 2.2 (pixel value of 186 for all R, G and B channels) was selected. The application gathers personal information provided by the viewers before the experiments starts. During the experiment, each test image is displayed simultaneously in two different sizes, one at the original size (i.e. 744 (v) x 560 (h)) and the other equivalent to the size of the built-in LCDs in each capturing device. Test images are displayed in random order and in random positions, one on the left side and the other on the right side of the display (i.e. left: large image, right: small image OR the opposite). The application automatically writes the observation data and saves them as a text file. The display interface is illustrated in Figure 1.

\subsection{Rank order method}

The display device was placed in a totally dark environment to prevent viewing flare. Rank order tests were conducted by displaying the same image at two different displayed sizes, side by side (Figure 1). Each observer took the test four times: for each camera, they judged the achromatic versions and the chromatic versions of the test stimuli.

Observers were placed $60 \mathrm{~cm}$ away from the display and were asked to adjust their observation distance, if necessary. They were asked to rank-order the attributes that were affected with changes in displayed image size. For the achromatic version of the stimuli they ranked the following attributes (from 1, the most affected to 4, the least affected): contrast, brightness, sharpness and noisiness. For the chromatic versions, in addition to the previous attributes, they also rankordered hue and colourfulness (rank order from 1 to 6). A total of 19 observers, 9 females and 8 males took the experiment. Their age ranged between 20 and 60 years old. They had mostly imaging and design backgrounds.

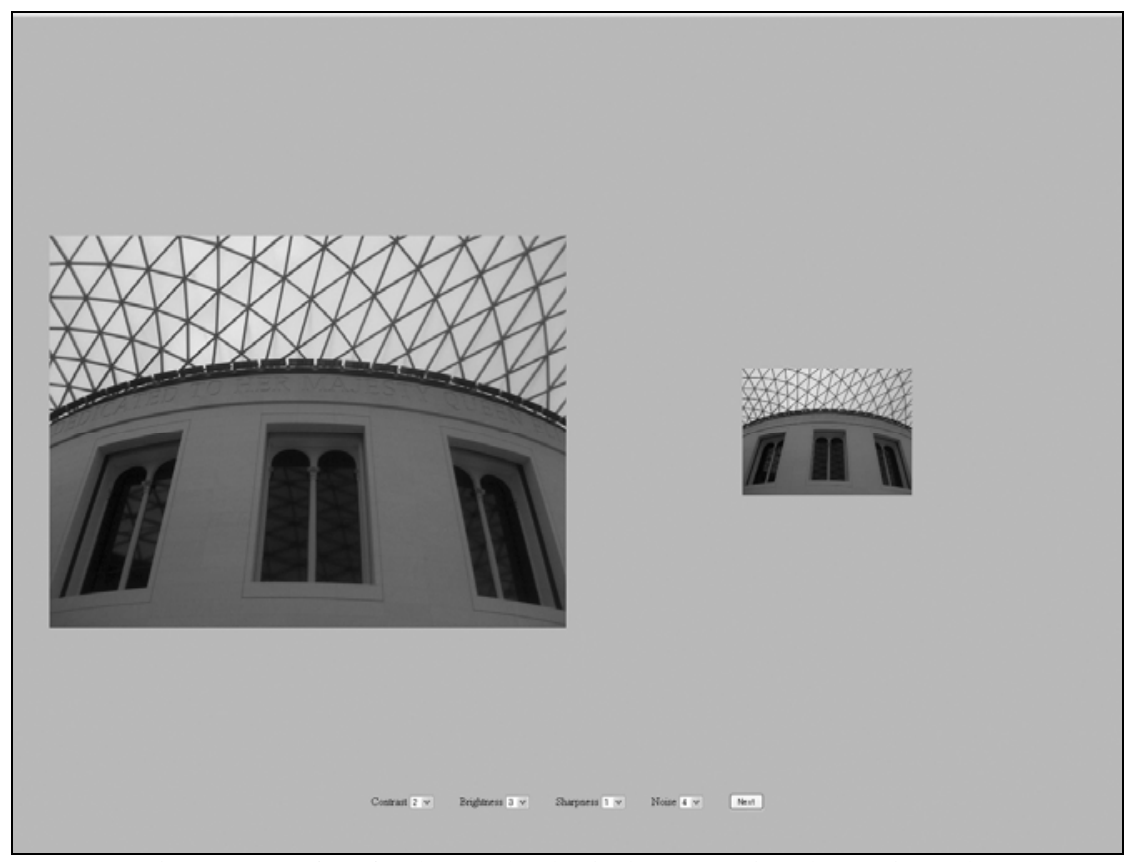

Figure 1. Interface for the psychophysical test page in achromatic mode. 


\section{RESULTS}

\subsection{Classification of test images}

The original full size versions of the test stimuli were categorised by both an objective method suggested by Triantaphillidou et al [1] and visual inspection. Triantaphillidou et al have suggested a method to analyse and classify scenes by deriving certain scene metric values that identify how much or how little of a 'scene characteristic' they may process. To discuss the proximity of the scene metric values between the different scenes and therefore the similarity in their characteristics, they classified these scene metrics into four ranges, ordered by relative distance from the median. Values found to be within one sigma from the median value were considered 'average' and were split into 'average-tolow', if below the median and 'average-to-high', if above the median. Another two categories included 'extreme' values, i.e. values that were more than plus or minus one standard deviation away from the median, comprising the 'very low' category if more than one sigma below the median, and 'very high' if more than one sigma above the median.

To investigate the relationships between the attribute ranking and the test-scene content, the test stimuli in this work were categorised according to five different image characteristics: three characteristics were related to both scene content and system performance and two characteristics were related only to the system performance. For examining the lightness (i.e. how light/dark they may be) and the colourfulness (i.e. how colourful/non-colourful they may be) characteristics of the test images, the original stimuli were categorised by both the suggested objective scene analysis method and visual inspection. For examining the busyness, sharpness and noisiness of the original test stimuli, only visual inspection in a dark surround was used.

Table 1 shows the selected image characteristics and the number of images that fall in each category, for both the Canon 30D and the Apple iPhone cameras. For the 'light/dark' characteristic, the test images obtained by both devices were similarly classified - similar number of images in each category. However, for the 'colourful/non-colourful' characteristic, the images were classified differently for each device due to differences in system performances. For example, in the case of colourfulness, some images from the Apple iPhone camera were objectively classified as 'colourful' due to high colour noise levels, even if they were not otherwise colourful in appearance. Also, more 'moderately dark' images captured with the Apple iPhone camera were categorised as 'non colourful' than when these captured with the Canon 30D.

The test images were categorised for the last three characteristics of busyness, sharpness and noisiness by visual inspection. Images possessing high amount of detail and texture were categorised as 'busy' whereas these possessing slow varying areas were categorised as 'non busy'. Images in-focus and/or possessing sharp edges were categorised as 'sharp' and these that appeared out-of-focus and/or possessing blurred and moving objects and/or where camera shake had been introduced, were categorised as 'un-sharp'. Images appeared to possess any visible noise were categorised as 'noisy'. The numbers of images classified into each category for the last two characteristics were different for the two camera systems, because the sharpness and the noisiness are highly dependent on system performance.

\begin{tabular}{|c|c|c|c|c|c|c|c|c|c|c|c|c|c|c|}
\hline & \multicolumn{4}{|c|}{ Dark/Light } & \multicolumn{4}{|c|}{ Colourful } & \multicolumn{2}{|c|}{ Busy } & \multicolumn{2}{|c|}{ Sharp } & \multicolumn{2}{|c|}{ Noisy } \\
\hline & Dark & $\begin{array}{c}\text { Moderately } \\
\text { dark }\end{array}$ & $\begin{array}{c}\text { Moderately } \\
\text { light }\end{array}$ & Light & Colourful & $\begin{array}{c}\text { Moderately } \\
\text { colourful }\end{array}$ & $\begin{array}{c}\text { Moderately } \\
\text { non colourful }\end{array}$ & $\begin{array}{c}\begin{array}{c}\text { Non } \\
\text { colourful }\end{array} \\
\end{array}$ & Yes & No & Yes & No & Yes & No \\
\hline $\begin{array}{c}\text { Canon } \\
\text { 30D }\end{array}$ & 8 & 24 & 22 & 10 & 17 & 15 & 21 & 11 & \multirow{2}{*}{30} & \multirow{2}{*}{34} & 49 & 15 & 30 & 34 \\
\hline $\begin{array}{c}\text { Apple } \\
\text { iPhone }\end{array}$ & 8 & 24 & 21 & 11 & 21 & 11 & 11 & 21 & & & 43 & 21 & 62 & 2 \\
\hline
\end{tabular}

Table 1. Numbers of images classified in each category according to their scene characteristics/properties and visual inspection.

\subsection{Results from attribute ranking}

The ranked scale data for all test stimuli were averaged for each mode (achromatic and chromatic) and each camera. Tables 2 and 3 present the average ranks for each attribute and these ranks also being converted to an ordinal scale (from 1 to 4 , or 1 to 6 ) that represents the most to the least affected attribute. For the achromatic version of the stimuli, the maximum average rank was 4.6 was the maximum average rank for the chromatic version of the stimuli. Attributes with higher average ranks were more affected and those with lower average ranks. 


\begin{tabular}{|c|c|c|c|c|c|c|c|c|c|c|c|c|}
\hline \multirow{2}{*}{\multicolumn{3}{|c|}{ Canon 30D }} & \multicolumn{4}{|c|}{ Achromatic } & \multicolumn{6}{|c|}{ Chromatic } \\
\hline & & & \multirow{2}{*}{$\begin{array}{c}\text { Contrast } \\
2.74 \\
\end{array}$} & \multirow{2}{*}{$\begin{array}{c}\text { Brightness } \\
2.30\end{array}$} & \multirow{2}{*}{$\begin{array}{c}\text { Sharpness } \\
2.78\end{array}$} & \multirow{2}{*}{$\begin{array}{c}\text { Noisiness } \\
2.18\end{array}$} & \multirow{2}{*}{$\begin{array}{c}\text { Contrast } \\
4.18 \\
\end{array}$} & \multirow{2}{*}{$\begin{array}{c}\text { Brightness } \\
3.61\end{array}$} & \multirow{2}{*}{$\begin{array}{c}\text { Sharpness } \\
4.65\end{array}$} & \multirow{2}{*}{\begin{tabular}{|c|} 
Noisiness \\
2.91 \\
\end{tabular}} & \multirow{2}{*}{$\begin{array}{l}\text { Hue } \\
2.52\end{array}$} & \multirow{2}{*}{$\begin{array}{c}\text { Colourfulness } \\
3.13\end{array}$} \\
\hline \multirow{8}{*}{ Dark } & \multirow{2}{*}{ Dark } & Average & & & & & & & & & & \\
\hline & & Order & 2 & 3 & 1 & 4 & 2 & 3 & 1 & 5 & 6 & 4 \\
\hline & \multirow{2}{*}{ Moderately dark } & Average & 2.73 & 2.24 & 3.05 & 1.98 & 4.10 & 3.64 & 4.66 & 2.42 & 2.65 & 3.52 \\
\hline & & Order & 2 & 3 & 1 & 4 & 2 & 3 & 1 & 6 & 5 & 4 \\
\hline & \multirow{2}{*}{$\begin{array}{c}\text { Moderately } \\
\text { bright }\end{array}$} & Average & 2.78 & 2.36 & 3.00 & 1.85 & 4.20 & 3.74 & 4.62 & 2.21 & 2.71 & 3.52 \\
\hline & & Order & 2 & 3 & 1 & 4 & 2 & 3 & 1 & 6 & 5 & 4 \\
\hline & \multirow{2}{*}{ Bright } & Average & 2.63 & 2.44 & 3.04 & 1.89 & 4.07 & 3.86 & 4.71 & 2.43 & 2.65 & 3.29 \\
\hline & & Order & 2 & 3 & 1 & 4 & 2 & 3 & 1 & 6 & 5 & 4 \\
\hline \multirow{8}{*}{ Colourful } & \multirow{2}{*}{ Colourful } & Average & 2.66 & 2.40 & 3.03 & 1.92 & 4.00 & 3.75 & 4.53 & 2.24 & 2.62 & 3.87 \\
\hline & & Order & 2 & 3 & 1 & 4 & 2 & 4 & 1 & 6 & 5 & 3 \\
\hline & \multirow{2}{*}{$\begin{array}{l}\text { Moderately } \\
\text { colourful }\end{array}$} & Average & 2.76 & 2.20 & 2.97 & 2.08 & 4.16 & 3.62 & 4.66 & 2.53 & 2.55 & 3.48 \\
\hline & & Order & 2 & 3 & 1 & 4 & 2 & 3 & 1 & 6 & 5 & 4 \\
\hline & \multirow{2}{*}{$\begin{array}{l}\text { Moderately non } \\
\text { colourful }\end{array}$} & Average & 2.74 & 2.32 & 3.01 & 1.93 & 4.17 & 3.73 & 4.72 & 2.43 & 2.73 & 3.22 \\
\hline & & Order & 2 & 3 & 1 & 4 & 2 & 3 & 1 & 6 & 5 & 4 \\
\hline & \multirow{2}{*}{ Non colourful } & Average & 2.82 & 2.38 & 2.96 & 1.84 & 4.28 & 3.70 & 4.70 & 2.48 & 2.72 & 3.12 \\
\hline & & Order & 2 & 3 & 1 & 4 & 2 & 3 & 1 & 6 & 5 & 4 \\
\hline \multirow{4}{*}{ Busy } & \multirow{2}{*}{ Yes } & Average & 2.76 & 2.31 & 3.03 & 1.90 & 4.11 & 3.63 & 4.69 & 2.33 & 2.67 & 3.58 \\
\hline & & Order & 2 & 3 & 1 & 4 & 2 & 3 & 1 & 6 & 5 & 4 \\
\hline & \multirow{2}{*}{ No } & Average & 2.71 & 2.33 & 2.97 & 1.99 & 4.17 & 3.77 & 4.63 & 2.48 & 2.65 & 3.31 \\
\hline & & Order & 2 & 3 & 1 & 4 & 2 & 3 & 1 & 6 & 5 & 4 \\
\hline \multirow{4}{*}{ Sharp } & Yes & Average & 2.76 & 2.36 & 2.95 & 1.93 & 4.17 & 3.69 & 4.63 & 2.38 & 2.66 & 3.48 \\
\hline & res & Order & 2 & 3 & 1 & 4 & 2 & 3 & 1 & 6 & 5 & 4 \\
\hline & No & Average & 2.67 & 2.21 & 3.14 & 1.99 & 4.06 & 3.73 & 4.74 & 2.53 & 2.66 & 3.28 \\
\hline & NO & Order & 2 & 3 & 1 & 4 & 2 & 3 & 1 & 6 & 5 & 4 \\
\hline & Yes & Average & 2.67 & 2.27 & 3.01 & 2.05 & 4.09 & 3.65 & 4.68 & 2.62 & 2.57 & 3.39 \\
\hline Noisy & res & Order & 2 & 3 & 1 & 4 & 2 & 3 & 1 & 5 & 6 & 4 \\
\hline No1sy & & Average & 2.79 & 2.37 & 2.99 & 1.86 & 4.19 & 3.75 & 4.63 & 2.22 & 2.74 & 3.48 \\
\hline & No & Order & 2 & 3 & 1 & 4 & 2 & 3 & 1 & 6 & 5 & 4 \\
\hline Ave & age ranks from all s & muli & 2.74 & 2.32 & 3.00 & 1.95 & 4.14 & 3.70 & 4.65 & 2.41 & 2.66 & 3.43 \\
\hline & Overall Order & & 2 & 3 & 1 & 4 & 2 & 3 & 1 & 6 & 5 & 4 \\
\hline
\end{tabular}

Table 2. Average ranks and rank order of the image attributes from the image stimuli obtained by the Canon 30D 


\begin{tabular}{|c|c|c|c|c|c|c|c|c|c|c|c|c|}
\hline \multirow{2}{*}{\multicolumn{3}{|c|}{ Apple iPhone }} & \multicolumn{4}{|c|}{ Achromatic } & \multicolumn{6}{|c|}{ Chromatic } \\
\hline & & & \multirow{2}{*}{$\begin{array}{c}\text { Contrast } \\
2.75\end{array}$} & \multirow{2}{*}{$\frac{\text { Brightness }}{2.16}$} & \multirow{2}{*}{$\begin{array}{c}\text { Sharpness } \\
2.52\end{array}$} & \multirow{2}{*}{$\begin{array}{c}\text { Noisiness } \\
2.57\end{array}$} & \multirow{2}{*}{$\begin{array}{c}\text { Contrast } \\
3.52\end{array}$} & \multirow{2}{*}{$\begin{array}{c}\text { Brightness } \\
3.23\end{array}$} & \multirow{2}{*}{$\begin{array}{c}\text { Sharpness } \\
4.07\end{array}$} & \multirow{2}{*}{$\begin{array}{c}\text { Noisiness } \\
4.20\end{array}$} & \multirow{2}{*}{$\begin{array}{l}\text { Hue } \\
2.91\end{array}$} & \multirow{2}{*}{$\begin{array}{c}\text { Colourfulness } \\
3.09\end{array}$} \\
\hline \multirow{8}{*}{ Dark } & \multirow{2}{*}{ Dark } & Average & & & & & & & & & & \\
\hline & & Order & 1 & 4 & 3 & 2 & 3 & 4 & 2 & 1 & 6 & 5 \\
\hline & \multirow{2}{*}{ Moderately dark } & Average & 2.64 & 2.26 & 2.95 & 2.15 & 3.71 & 3.43 & 4.27 & 3.34 & 2.88 & 3.37 \\
\hline & & Order & 2 & 3 & 1 & 4 & 2 & 3 & 1 & 5 & 6 & 4 \\
\hline & \multirow{2}{*}{$\begin{array}{c}\text { Moderately } \\
\text { bright }\end{array}$} & Average & 2.69 & 2.17 & 3.04 & 2.10 & 3.51 & 3.29 & 4.45 & 3.29 & 2.98 & 3.48 \\
\hline & & Order & 2 & 3 & 1 & 4 & 2 & 4 & 1 & 5 & 6 & 3 \\
\hline & \multirow{2}{*}{ Bright } & Average & 2.59 & 2.18 & 3.02 & 2.21 & 3.41 & 3.20 & 4.45 & 3.67 & 2.96 & 3.31 \\
\hline & & Order & 2 & 4 & 1 & 3 & 3 & 5 & 1 & 2 & 6 & 4 \\
\hline \multirow{8}{*}{ Colourful } & \multirow{2}{*}{ Colourful } & Average & 2.70 & 2.13 & 3.03 & 2.14 & 3.47 & 3.39 & 4.30 & 3.13 & 2.99 & 3.71 \\
\hline & & Order & 2 & 4 & 1 & 3 & 3 & 4 & 1 & 5 & 6 & 2 \\
\hline & \multirow{2}{*}{$\begin{array}{l}\text { Moderately } \\
\text { colourful }\end{array}$} & Average & 2.67 & 2.24 & 2.94 & 2.16 & 3.71 & 3.33 & 4.35 & 3.22 & 2.82 & 3.57 \\
\hline & & Order & 2 & 3 & 1 & 4 & 2 & 4 & 1 & 5 & 6 & 3 \\
\hline & \multirow{2}{*}{$\begin{array}{l}\text { Moderately non } \\
\text { colourful }\end{array}$} & Average & 2.70 & 2.12 & 2.90 & 2.28 & 3.59 & 3.35 & 4.56 & 3.79 & 2.62 & 3.10 \\
\hline & & Order & 2 & 4 & 1 & 3 & 3 & 4 & 1 & 2 & 6 & 5 \\
\hline & \multirow{2}{*}{ Non colourful } & Average & 2.61 & 2.30 & 2.87 & 2.23 & 3.59 & 3.22 & 4.24 & 3.83 & 3.09 & 3.03 \\
\hline & & Order & 2 & 3 & 1 & 4 & 3 & 4 & 1 & 2 & 5 & 6 \\
\hline \multirow{4}{*}{ Busy } & \multirow{2}{*}{ Yes } & Average & 2.70 & 2.19 & 3.05 & 2.05 & 3.63 & 3.47 & 4.38 & 3.06 & 2.93 & 3.52 \\
\hline & & Order & 2 & 3 & 1 & 4 & 2 & 4 & 1 & 5 & 6 & 3 \\
\hline & \multirow{2}{*}{ No } & Average & 2.63 & 2.21 & 2.84 & 2.32 & 3.51 & 3.18 & 4.29 & 3.86 & 2.93 & 3.22 \\
\hline & & Order & 2 & 4 & 1 & 3 & 3 & 5 & 1 & 2 & 6 & 4 \\
\hline \multirow{4}{*}{ Sharp } & Yes & Average & 2.70 & 2.22 & 2.92 & 2.16 & 3.57 & 3.33 & 4.28 & 3.44 & 3.01 & 3.36 \\
\hline & & Order & 2 & 3 & 1 & 4 & 2 & 5 & 1 & 3 & 6 & 4 \\
\hline & No & Average & 2.60 & 2.17 & 2.97 & 2.26 & 3.57 & 3.29 & 4.44 & 3.58 & 2.77 & 3.37 \\
\hline & 100 & Order & 2 & 4 & 1 & 3 & 3 & 5 & 1 & 2 & 6 & 4 \\
\hline & Yoc & Average & 2.66 & 2.20 & 2.94 & 2.21 & 3.55 & 3.31 & 4.34 & 3.53 & 2.93 & 3.35 \\
\hline Noisy & res & Order & 2 & 4 & 1 & 3 & 2 & 5 & 1 & 3 & 6 & 4 \\
\hline & No & Average & 2.91 & 2.29 & 2.94 & 1.85 & 4.22 & 3.59 & 4.28 & 2.25 & 3.03 & 3.63 \\
\hline & 100 & Order & 2 & 3 & 1 & 4 & 2 & 4 & 1 & 6 & 5 & 3 \\
\hline Ave & gge ranks from all & muli & 2.66 & 2.20 & 2.94 & 2.19 & 3.57 & 3.32 & 4.33 & 3.49 & 2.93 & 3.36 \\
\hline & Overall Order & & 2 & 3 & 1 & 4 & 2 & 5 & 1 & 3 & 6 & 4 \\
\hline
\end{tabular}

Table 3. Average ranks and rank order of the image attributes from the image stimuli obtained by the Apple iPhone camera. 


\section{DISCUSSION}

Figure 2 shows the average ranks for all images captured by both cameras. The higher the ranking the more affected the attribute.

Overall, for the achromatic version of the stimuli obtained by both cameras, observers ranked sharpness as the most affected and contrast as the second most affected attributes with changes in the displayed image size. Brightness and noisiness were ranked as the third and fourth, respectively. For the stimuli obtained by the Apple iPhone camera, the observers also ranked brightness and noisiness as third and fourth respectively, but with a smaller difference in average ranks (2.19 and 2.20).

For the chromatic version of the stimuli captured with the Canon 30D, observers again ranked sharpness, contrast, and brightness in the same order as for the achromatic stimuli. Colourfulness and hue were found to be affected less, whilst noisiness was the least affected.

For the chromatic version of stimuli from the Apple iPhone camera, observers again ranked sharpness and contrast as the first two most affected attributes. However, noisiness, colourfulness and brightness were ranked as third, fourth and fifth whilst hue was ranked last.

The ranking of the attributes for the chromatic version of stimuli from the Apple iPhone camera was different, with noise and colourfulness having higher ranks, compared to the Canon 30D. This result is related to the relatively lower average lightness and the higher level of chromatic noise that were presented in most of the test stimuli originating from the Apple iPhone camera.

Overall rank-orders of the attributes are as below:

Achromatic stimuli:

Sharpness $>$ Contrast $>$ Brightness $>$ Noisiness

(Both devices)

Chromatic stimuli:

Sharpness $>$ Contrast $>$ Brightness $>$ Colourfulness $>$ Hue $>$ Noisiness (Canon 30D)

Sharpness $>$ Contrast $>$ Noisiness $>$ Colourfulness $>$ Brightness $>$ Hue (Apple iPhone)

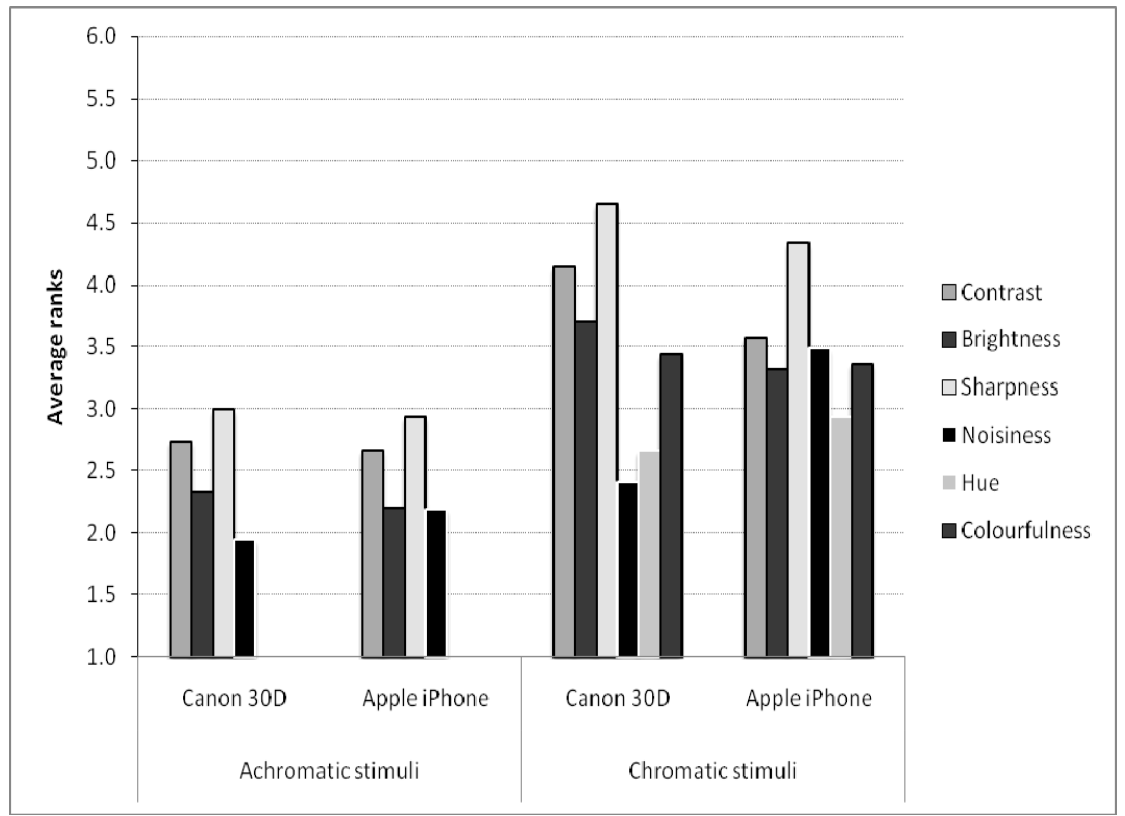

Figure 2. Average ranks from all test stimuli. 
Figures 3 to 7 present the average ranks in relation to image categories that were described in section 4.1.

In general, test stimuli categorised as 'dark', thus contain higher level of noise, sharpness was affected less and noisiness was affected more compared with other stimuli classified in different categories (figure 3). For test stimuli obtained by the Canon 30D, the average ranks for brightness was slightly increased as the average lightness of the test stimuli increased (figure 3). In a similar fashion, the average ranks of colourfulness was increased with 'colourful' stimuli and decreased with 'non colourful' stimuli (figure 4).

Average ranks of noisiness were decreased with the stimuli categorised as 'busy' whilst that with stimuli categorised as 'non busy' they were increased (figure 5). This is because of the noise being masked by the high frequency information in the scene. Average ranks of contrast were increased when stimuli categorised as 'sharp', whilst that of sharpness and noisiness were decreased (figure 6). The noise level of the test stimuli were also related to hue and colourfulness in the chromatic versions (figure 7). Average ranks of noisiness were affected more with stimuli categorised as 'noisy', indicating that when noise is visible in the image then noisiness becomes an important attribute with respect to changes in image size (figure 7).

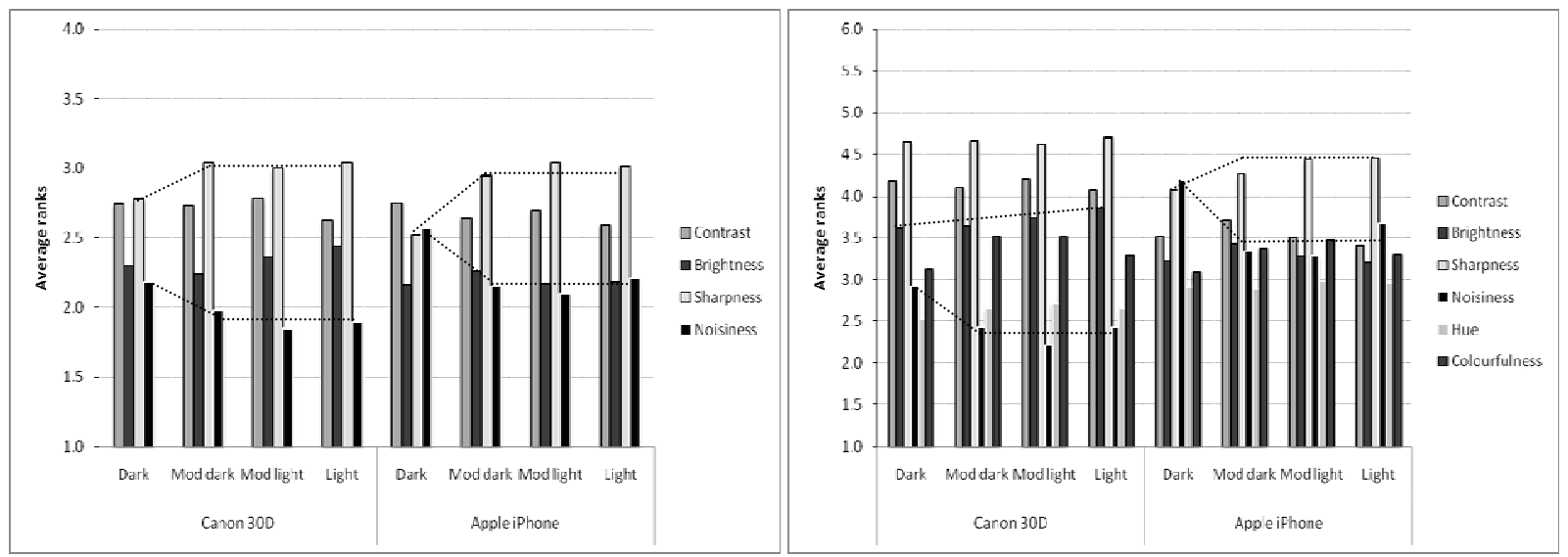

Figure 3. Average ranks of the image attributes of test stimuli categorised by their average lightness. Results from the achromatic versions of stimuli (left) and from the chromatic versions of stimuli (right).

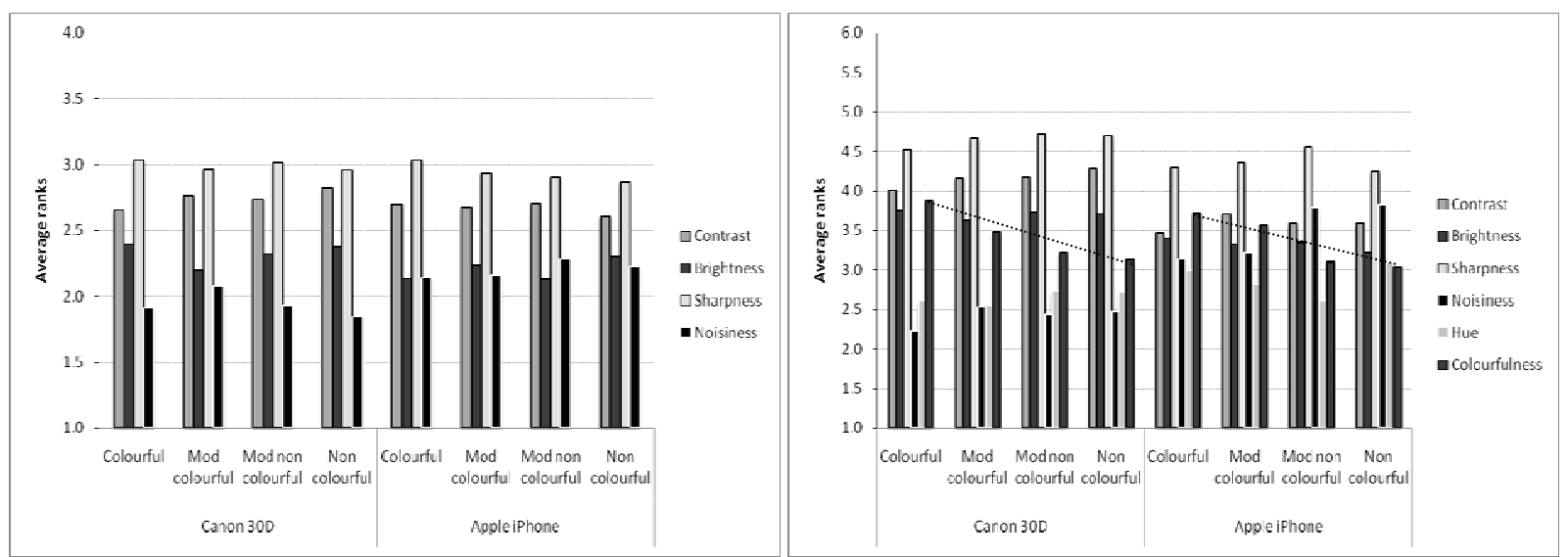

Figure 4. Average ranks of the image attributes of test stimuli categorised by their colourfulness. Results from the achromatic versions of stimuli (left) and from the chromatic versions of stimuli (right). 


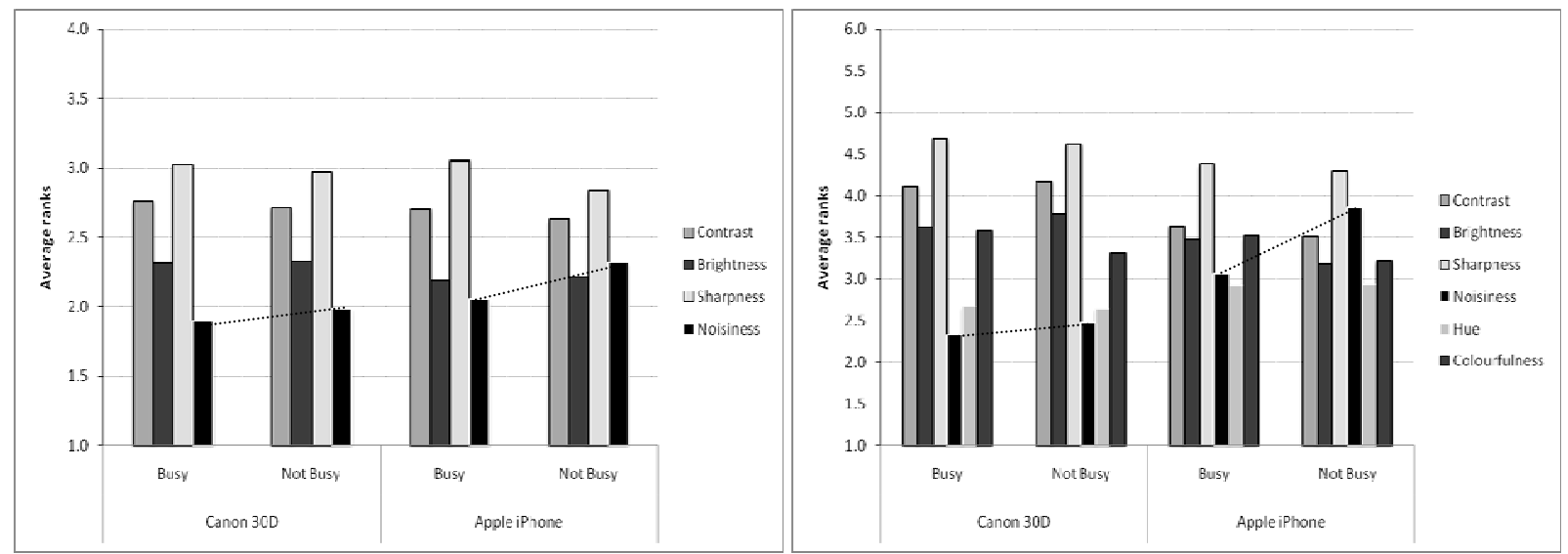

Figure 5. Average ranks of the image attributes of test stimuli categorised by their busyness. Results from the achromatic versions of stimuli (left) and from the chromatic versions of stimuli (right).

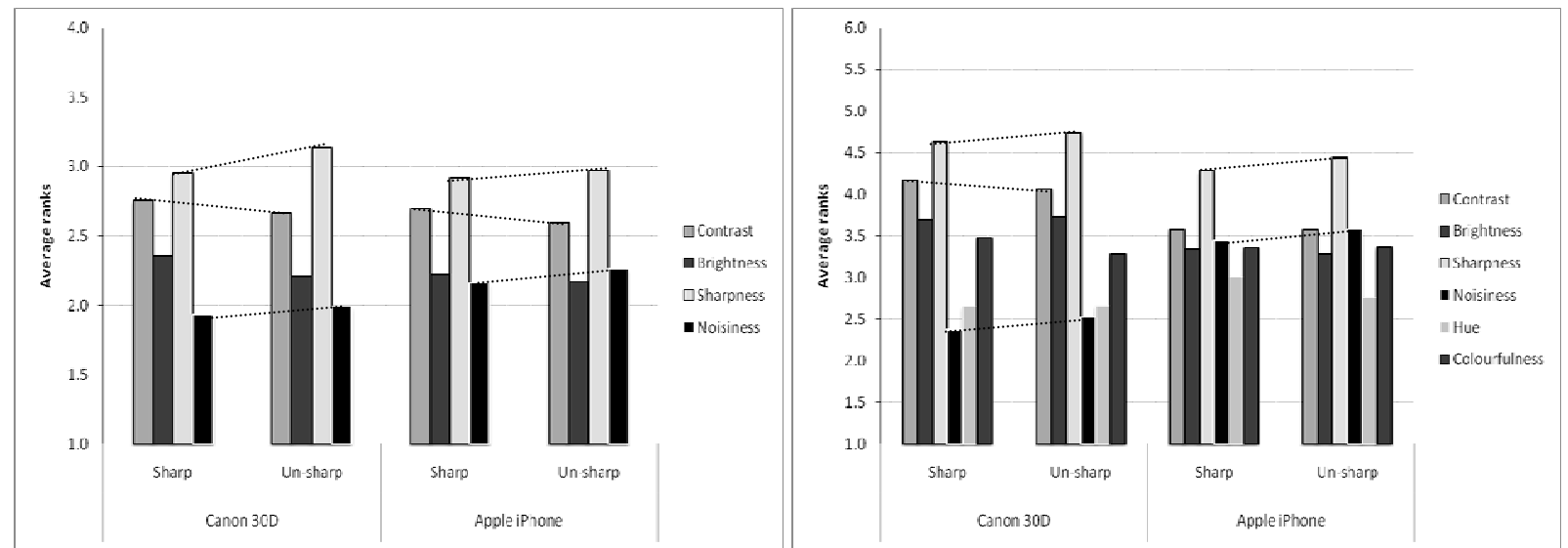

Figure 6. Average ranks of the image attributes of test stimuli categorised by their sharpness. Results from the achromatic versions of stimuli (left) and from the chromatic versions of stimuli (right).

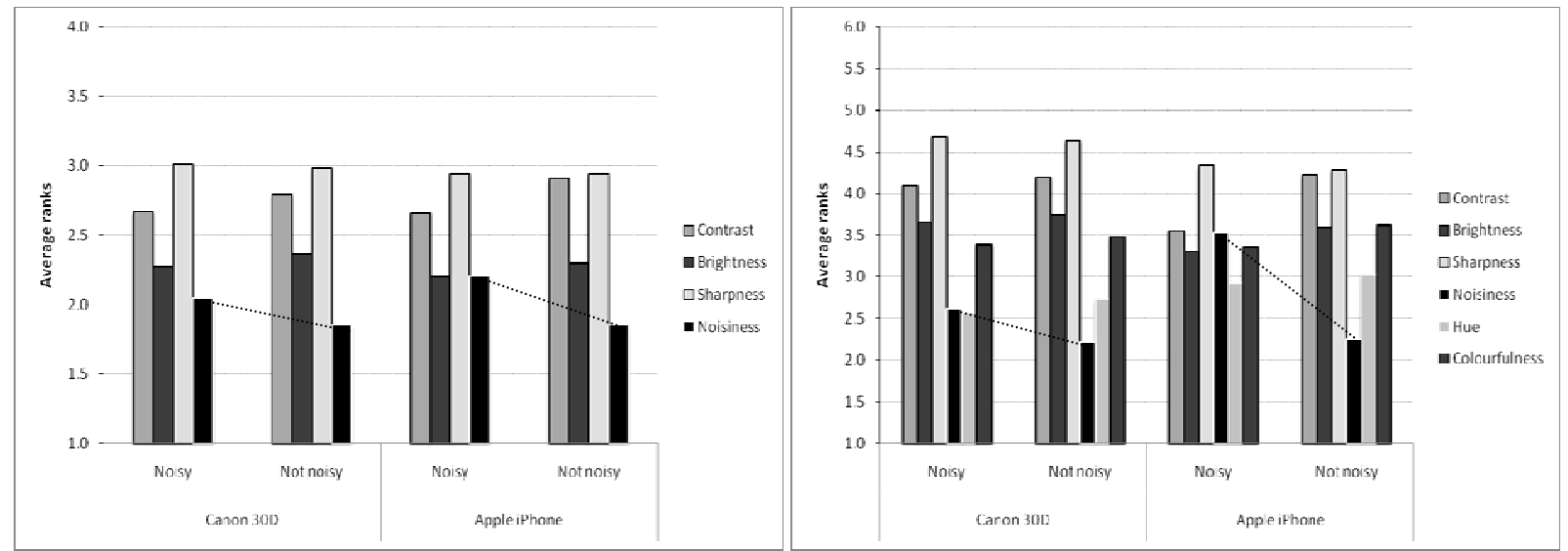

Figure 7. Average ranks of the image attributes of test stimuli categorised by their noise level. Results from the achromatic versions of stimuli (left) and from the chromatic versions of stimuli (right). 


\section{CONCLUSIONS}

A psychophysical experiment was carried out to investigate the changes in image appearance when images are viewed at different image sizes. Two sets of digital capturing devices with different overall quality were used to record 64 natural scenes with various scene content under various illumination conditions. Six image attributes were investigated. In addition, the relationships between most affected attributes and scene characteristics were investigated.

Results from the achromatic stimuli obtained by both capturing devices indicated that, observers found that sharpness is the most affected image attribute with changes in displayed image size, then contrast and then brightness. Noisiness was ranked as the least affected attribute for the stimuli obtained by both devices. However, from the chromatic versions of the stimuli, results from the two capturing devices did not always match. Although sharpness and contrast were again the first two attributes affected by the changes in size, brightness did not always come third. Observers also found that colourfulness was the 4th most affected attribute for the images obtained by both devices.

The results varied with scene characteristics. The average lightness level of a scene was found to be related to the attributes of sharpness and noisiness, whilst the colourfulness of a scene was found to be related more to the attribute of colourfulness. The busyness of a scene was found to be also related to noisiness, i.e. as business increased noisiness became less and less important. The sharpness of a scene was related to the attributes of sharpness and noisiness whilst the noise level of a scene was related to noisiness.

Further research work is currently taking place to model the changes in image sharpness with changes in displayed image size using various sharpness matching techniques.

\section{REFERENCES}

[1] Triantaphillidou, S., Allen, E., Jacobson, R., E., "Image quality comparison between JPEG and JPEG2000, Part IIScene Dependency, Scene Analysis and Classification”, J. of Imaging Science and Technology, 51(3), 259-270 (2007).

[2] Day, E., A., Taplin, L., Berns, R., S., "Colorimetric characterization of a computer-controlled liquid crystal display", Color Research and Application, 29(5), 365-373 (2004).

[3] Berns, R., S., “Methods for characterizing CRT displays”, Displays, 16(4), 173-182 (1996).

[4] Triantaphillidou, S., Jacobson, R., E., "Measurements of the modulation transfer function of image displays", J. of Imaging Science and Technology, 48 (1), 58-65 (2004).

[5] MacDonald, L. W. and Luo, M. R., [Colour Imaging: Vision and Technology], John Wiley \& Sons Ltd, Chichester, Chapter 15, (1999).

[6] Fairchild, M. D., [Color Appearance Models, 2nd ed.], John Wiley \& Sons Ltd, Chichester, Chapter 20 (2005).

[7] Nezamabadi, M., Berns, R. S., "The effect of image size on the color appearance of image reproductions using colorimetrically calibrated LCD and DLP displays”, J. of Society for Information Display, 14(9), 773-783 (2005).

[8] Choi, S. Y., Luo, M. R., Pointer, M. R., "Colour Appearance change of a large size display under various illumination conditions”, Proc. SPIE/IS\&T Electronic Imaging 6493, 6493081-6483089 (2007).

[9] Nezamabadi, M., Montag, E. D., Berns, R. S., "An investigation of the effect of image size on the color appearance of softcopy reproductions using a contrast matching technique”, SPIE/IS\&T Electronic Imaging 6493, 64930916493099 (2007).

[10] Engeldrum, P. G., [Psychometric Scaling], Imcotek Press, Winchester, Chapter 6 (2000). 